\title{
Accuracy of clinical diagnosis of idiopathic Parkinson's disease: a clinico-pathological study of 100 cases
}

\author{
Andrew J Hughes, Susan E Daniel, Linda Kilford, Andrew J Lees
}

\begin{abstract}
Few detailed clinico-pathological correlations of Parkinson's disease have been published. The pathological findings in 100 patients diagnosed prospectively by a group of consultant neurologists as having idiopathic Parkinson's disease are reported. Seventy six had nigral Lewy bodies, and in all of these Lewy bodies were also found in the cerebral cortex. In 24 cases without Lewy bodies, diagnoses included progressive supranuclear palsy, multiple system atrophy, Alzheimer's disease, Alzheimer-type pathology, and basal ganglia vascular disease. The retrospective application of recommended diagnostic criteria improved the diagnostic accuracy to $82 \%$. These observations call into question current concepts of Parkinson's disease as a single distinct morbid entity.
\end{abstract}

Necropsy studies of patients with Parkinson's syndrome show that idiopathic Parkinson's disease (IPD) comprises between 60 and $75 \%$ of cases. ${ }^{12}$ In life most of these are correctly diagnosed, but despite the application of stringent diagnostic criteria ${ }^{3}$ misdiagnoses do occur, with some patients thought clinically to have IPD turning out to have other illnesses. $^{124}$ Conversely, in other patients with atypical clinical pictures the diagnosis of IPD is established only after death. ${ }^{56}$

There are few clinico-pathological studies from which to assess the accuracy of clinical diagnosis but it is estimated at around $80 \% .^{27}$ Multiple system atrophy, progressive supranuclear palsy, Alzheimer's disease, and cerebrovascular pathology are thought to make up most misdiagnoses. ${ }^{124}$

The Parkinson's Disease Society Brain Bank (PDSBB) in London receives donor tissue from parkinsonian patients, most of whom have been prospectively examined annually by a neurologist. To assess the accuracy of clinical diagnosis we examined the neuropathology in 100 patients who were thought to have IPD. For patients in whom the diagnosis was histologically substantiated the clinical variables were correlated with the pathological findings.

\section{Materials and methods}

We studies 100 consecutive cases with clinically diagnosed IPD. The brains were collected over a three year period between June 1987 and August 1990 from all over the United
Kingdom. Seventy cases were registered donors and had been prospectively assessed annually by neurologists associated with the PDSBB. All other cases had been diagnosed by consultant neurologists or geriatricians as having IPD. Hospital and consultant case notes were reviewed to supplement clinical information. Age at onset of illness, disease duration, initial response to levodopa, and severity of disease at death, as measured by the Hoehn and Yahr score, ${ }^{8}$ were correlated with the pathological findings.

Half brains fixed in $10 \%$ neutral formalin were available for examination by standard neuropathological methods. Tissue for paraffin embedding was taken from the cerebral cortex, striatum, midbrain, pons, and medulla. In most cases all areas of cortex (frontal, temporal, parietal, occipital) and the cerebellum (hemisphere and vermis) were examined. Sections were stained with haematoxylin-eosin (HE), luxol fast blue cresyl-violet, and modified Bielschowsky silver impregnation. On selected regions, immunocytochemistry was performed with biotin-streptavidin method and antibody to ubiquitin (Dako, polyclonal 1:400).

The diagnosis of IPD was based on finding a clear depletion of brain stem pigmented neurones with Lewy bodies in some of the remaining nerve cells. ${ }^{9}$ In all cases where Lewy bodies were difficult to find, at least three HE stained $7 \mu$ sections of midbrain were examined. In each section there were usually more than 150 pigmented nigral neurones. The cerebral cortex was carefully screened for intraneuronal Lewy bodies with HE stained sections. In most cases the frontal anterior cingulate gyrus was used, however, in a few cases only the parahippocampal gyrus was available. Lewy bodies were considered to be found easily if identified within 5 minutes' inspection.

Sections were stained with anti-ubiquitin if Lewy bodies had not been identified after 10 minutes' observation time. Nerve cell loss in the substantia nigra and the locus coeruleus was assessed by two independent observers with a 4 point ( $1=$ mild, $4=$ severe $)$ grading system, which was based on comparison with age-matched controls. The presence of Lewy bodies in the locus coeruleus and dorsal vagal nucleus was recorded for each case.

In cases lacking the pathological changes of IPD, diagnoses were established by accepted neuropathological criteria. The criteria proposed by Khachaturian ${ }^{10}$ were used to identify cases in which neocortical senile plaques were numerous. This group was sub-divided into 
Alzheimer's disease and Alzheimer-type pathology. For the diagnosis of Alzheimer's disease the presence of numerous neocortical neurofibrillary tangles and a history of dementia were required. Cases with Alzheimer-type pathology had few or no neocortical tangles and no definite dementia. Progressive supranuclear palsy was classified as histologically typical $^{11}$ and atypical; the latter being distinguished by predominant pallido-luysionigral degeneration with neurofibrillary tangles, few brain stem tangles, and a normal dentate nucleus. Cases of multiple system atrophy ${ }^{4}$ showed striatonigral involvement combined in all the cases with some degree of olivopontocerebellar damage. Arteriolosclerosis with widening of perivascular spaces in the deep grey nuclei was considered a normal accompaniment of ageing. Vascular damage was considered important when there were ischaemic infarcts. A single case of postencephalitic parkinsonism ${ }^{1}$ was distinguished by severe nigral damage with widespread brain stem tangles in a patient with disease onset in 1955 , when aged 34 , having possibly been exposed to an encephalitic-like illness at the age of eight years.

Clinical variables were correlated with the neuropathological features with Spearman's rank correlation test. The clinical and pathological data from patient subgroups were compared with Student's $t$ test. The case histories of all patients were reviewed by two of us (AJL, $\mathrm{AJH})$ and the clinical diagnoses re-evaluated with the PDSBB clinical criteria ${ }^{3}$ (box).

\section{Results}

The mean age of disease onset was 64.5 (range 31-85) years, and the mean duration of disease was 11.9 (range 2-35) years; 59 patients were men and 41 were women. Seventy six cases

\footnotetext{
UK Parkinson's Disease Society Brain Bank clinical diagnostic criteria Step 1 Diagnosis of Parkinsonian syndrome

Bradykinesia (slowness of initiation of voluntary movement with progressive reduction in speed and amplitude of repetitive actions)

- And at least one of the following: muscular rigidity 4-6 $\mathrm{Hz}$ rest tremor

postural instability not caused by primary visual, vestibular, cerebellar, or proprioceptive dysfunction.

Step 2 Exclusion criteria for Parkinson's disease

History of repeated strokes with stepwise progression of parkinsonian features

- History of repeated head injury

- History of definite encephalitis

Oculogyric crises

Neuroleptic treatment at onset of symptoms

More than one affected relative

Sustained remission

- Strictly unilateral features after 3 years

Supranuclear gaze palsy

Cerebellar signs

Early severe autonomic involvement

Early severe dementia with disturbances of memory, language, and praxis

Babinski sign

Presence of cerebral tumour or communicating hydrocephalus on CT scan

Negative response to large doses of levodopa (if malabsorption excluded)

- MPTP exposure

Step 3 Supportive prospective positive criteria for Parkinson's disease

(Three or more required for diagnosis of definite Parkinson's disease)

Unilateral onset

Rest tremor present

Progressive disorder

Persistent asymmetry affecting side of onset most

Excellent response (70-100\%) to levodopa

- Severe levodopa-induced chorea

Levodopa response for 5 years or more

Clinical course of 10 years or more
}

fulfilled the pathological criteria for IPD, while 24 patients were clinically misdiagnosed. There was no significant difference between the two groups for age at onset, age at death, or terminal disease severity (table 1). The duration of disease was significantly longer in cases with IPD, mean 12.8 (range 2-30) years, compared to those with other diagnoses, mean 8.8 (range 2-35) years $(p=0.006)$. Where information was available, $96 \%(66 / 69)$ of those with pathologically confirmed IPD and $82 \%(18 / 22)$ of those without Lewy body pathology had a definite initial response to levodopa. After retrospective assessment of the clinical features 11 patients were judged not to fulfil the PDSBB clinical criteria for IPD. ${ }^{3}$ Nine had exclusion criteria for Parkinson's disease (table 1), including no response to an adequate trial of levodopa (4), early severe dementia (2), early autonomic failure (2), history of stepwise progression related to strokes (1), and supranuclear gaze palsy (1). The two others had inadequate supportive positive criteria (table 1). Eight of the 11 patients had pathological findings other than IPD while three had typical Lewy body pathology.

Neuropathological findings-Lewy bodies were found easily in the substantia nigra of most cases with histological IPD. In a few, where there was severe cell loss, it was necessary to examine more than one section before they were identified. Lewy bodies were also present in the locus coeruleus in $76 \%(54 / 71)$ and in the dorsal vagal nucleus in $79 \%(49 / 62)$ of cases where suitable tissue was available for examination. Cortical Lewy bodies were found in all cases with histological IPD, including seven patients in whom the disease duration was 5 years or less. In $58(76 \%)$ they were easily found; nine $(12 \%)$ required a prolonged search; a further nine (12\%) needed ubiquitin staining for identification. A formal study of Lewy body distribution in the different cortical areas was not undertaken. In most cases, however, we found that examination of the anterior cingulate gyrus yielded positive results most quickly. Only one case showed more than five Lewy bodies per field at $\times 100$ magnification, thereby satisfying the recently proposed criteria for diagnosis of diffuse Lewy body disease. $^{12}$ Cell loss in the substantia nigra, relative to age-matched controls, was estimated as mild in eight (11\%), mild to moderate in $23(30 \%)$, moderate to severe in 30 $(39 \%)$, and severe in $15(20 \%)$ of cases.

Co-existing striatal pathology was found in 27 (36\%) IPD brains; in most there was vascular damage with multiple lacunar infarcts (table 2). Pathology outside of the nigrostriatal system occurred in $19(22 \%)$ cases; in nine $(12 \%)$ there was co-existent Alzheimer's disease, seven (9\%) had vascular damage, and one case had Alzheimer-type pathology.

The principal findings in the 24 cases without Lewy bodies were progressive supranuclear palsy (six; two classical and four atypical), multiple system atrophy (five), $\mathrm{Alz}$ heimer's disease (three), Alzheimer-type 
Table 1 Clinical variables in patients with histologically confirmed Parkinson's disease and in those with other pathological diagnoses ${ }^{3}$

\begin{tabular}{llc}
\hline Variable & $\begin{array}{l}I P D \\
(n=76)\end{array}$ & $\begin{array}{l}\text { Non-IPD } \\
(n=24)\end{array}$ \\
\hline Mean (range) age at onset (years) & $63 \cdot 6(31-84)$ & $67 \cdot 6(34-85)$ \\
Mean (range) duration of disease (years) & $12 \cdot 8(2-30)$ & $8 \cdot 8(2-35)$ \\
Mean (range) Hoehn and Yahr score at death & $4 \cdot 3(3-5)$ & $4 \cdot 4(3-5)$ \\
Mean (range) age at death (years) & $76 \cdot 4(50-91)$ & $76 \cdot 6(62-90)$ \\
Initial levodopa response & & 2 \\
$\quad$ unknown & 7 & 4 \\
nil to poor & 3 & 2 \\
moderate & 9 & 12 \\
good & 37 & 4 \\
excellent & 20 & \\
\hline
\end{tabular}

pathology (three), vascular disease (three), isolated nigral atrophy with no Lewy bodies (two), and postencephalitic parkinsonism (one) (table 3 ). In one case there were no abnormal pathological findings and review of the clinical details raised the possibility of benign essential tremor.

Clinico-pathological correlations in the cases of IPD-For the 76 patients with histological IPD there was a positive correlation between the severity of nigral cell loss and both disease duration $(r=0.31 ; p=0.008)$ and severity at death $(r=0.39 ; p=0.001)$. There was also a positive correlation between the duration of disease and severity at death $(r=0.34 ; p=$ 0.003 ) and between cell loss in the substantia nigra and the locus coeruleus $(r=0.38 ; p=$

Table 2 Pathological findings in 76 cases with histologically confirmed Parkinson's disease

\begin{tabular}{|c|c|c|c|}
\hline \multirow{2}{*}{$\begin{array}{l}\text { Pathological findings } \\
\text { IPD with co-existent striatal pathology }\end{array}$} & \multicolumn{3}{|c|}{ No of cases } \\
\hline & & & 27 \\
\hline Vascular & & 19 & \\
\hline $\begin{array}{l}\text { lacunar infarction } \\
\text { putaminal haemorrhage } \\
\text { putaminal AVM }\end{array}$ & $\begin{array}{r}16 \\
2 \\
1\end{array}$ & & \\
\hline Striatal plaques & & 7 & \\
\hline $\begin{array}{l}\text { with neocortical involvement } \\
\text { isolated to striatum with normal } \\
\text { neocortex }\end{array}$ & $\begin{array}{l}5 \\
2\end{array}$ & & \\
\hline Caudate atrophy & & 1 & \\
\hline $\begin{array}{l}\text { IPD with pathology outside } \\
\text { nigro-striatal system }\end{array}$ & & & 19 \\
\hline $\begin{array}{l}\text { Alzheimer's disease } \\
\text { Alzheimer-type pathology }\end{array}$ & & 9 & \\
\hline Vascular events & & 7 & \\
\hline $\begin{array}{l}\text { cerebral infarction } \\
\text { cerebellar infarction } \\
\text { cerebellar haemorrhage }\end{array}$ & $\begin{array}{l}5 \\
1 \\
1\end{array}$ & & \\
\hline Abscess & & 1 & \\
\hline "Diffuse Lewy body disease" & & 1 & \\
\hline
\end{tabular}

See text for definitions of Alzheimer's disease. Alzheimer-typ pathology and diffuse Lewy body disease.

Table 3 Pathological findings in 24 cases clinically mis-diagnosed as Parkinson's disease

\begin{tabular}{|c|c|}
\hline Pathological findings & No of cases \\
\hline $\begin{array}{l}\text { Progressive supranuclear palsy } \\
\text { atypical } \\
\text { classical }\end{array}$ & $\begin{array}{l}4 \\
2\end{array}$ \\
\hline $\begin{array}{l}\text { Multiple system atrophy } \\
\text { Alzheimer's disease }\end{array}$ & $\begin{array}{l}5 \\
3\end{array}$ \\
\hline $\begin{array}{l}\text { with striatal involvement } \\
\text { without striatal involvement }\end{array}$ & $\begin{array}{l}1 \\
2\end{array}$ \\
\hline $\begin{array}{l}\text { Alzheimer-type pathology } \\
\text { with striatal involvement }\end{array}$ & 3 \\
\hline $\begin{array}{l}\text { Vascular, lacunar state } \\
\text { Nigral atrophy without Lewy bodies } \\
\text { Postencephalitic parkinsonism } \\
\text { Normal ?essential tremor }\end{array}$ & $\begin{array}{l}3 \\
2 \\
1 \\
1\end{array}$ \\
\hline
\end{tabular}

See text for definitions of Alzheimer's disease. Alzheimer-type pathology, progressive supranuclear palsy, multiple system atrophy, postencephalitic parkinsonism.
$0.002)$. In $54(71 \%)$ patients the cause of death was known. All correlations were greatest for the $46 \%(26 / 54)$ of patients who died from causes clearly unrelated to their IPD (for example, myocardial infarction, stroke, cancer). The $r$ value for the correlation between nigral cell loss and disease duration in this group increased to $0.52(p=0.01)$.

\section{Discussion}

The concept of Parkinson's disease is historically a clinical one stemming from James Parkinson's ${ }^{13}$ seminal description which was later embellished by the nineteenth century neurologists Charcot and Gowers. Many authorities have drawn attention to the difficulties in distinguishing Parkinson's disease clinically from other parkinsonian syndromes. Progress in this area has been hampered by the failure to apply rigid clinical criteria and sparse clinical documentation in published pathological series. ${ }^{1415}$ Furthermore, how much disease classification relied on pathological criteria such as severity of nigral cell loss or the presence of Lewy bodies, is unclear.

In our study $76 \%$ of cases satisfied the established neuropathological criteria for IPD. Despite the guideline PDSBB criteria for the clinical diagnosis of $\mathrm{IPD}^{3}$ those used by individual neurologists clearly differed. Nevertheless, each patient included in this study had been specifically considered during life to have IPD rather than a less well-defined parkinsonian syndrome. The retrospective application of the recommended diagnostic criteria ${ }^{3}$ improved the diagnostic accuracy to $82 \%$ $(73 / 89) ; 16$ of the 24 cases misdiagnosed, however, still fulfilled these criteria and three others with atypical clinical features had typical Lewy body pathology.

The occurrence of cortical Lewy bodies in all cases of IPD has not been previously reported. Our finding suggests the causative agent of IPD produces damage beyond the confines of those nuclei regularly reported as being involved-for example, substantia nigra, substantia innominata, locus coeruleus, dorsal vagal nucleus. We suggest that the widespread distribution of Lewy bodies may reflect a "field change" in certain neuronal types, one of which may be cells synthesising tyrosine hydroxylase, which have recently been identified in the cerebral cortex. ${ }^{16}$ Whether Lewy bodies are formed primarily at a specific sitefor example, the substantia nigra-with later development elsewhere is not known, but our findings of widespread Lewy bodies in patients with short disease duration suggest concurrent formation may occur.

An unexpected finding was the high incidence of Alzheimer's disease and Alzheimertype pathology which had been diagnosed clinically as IPD. Extrapyramidal signs in Alzheimer's disease are well reported and have in part been attributed to the severity of cell loss in the substantia nigra. ${ }^{17} 18$ In previous reports, however, it has been suggested that these patients are easily distinguishable from those with IPD. ${ }^{19}$ Our findings suggest that 
such a distinction cannot always be made. Furthermore, we wish to draw attention to those patients with a parkinsonian syndrome and Alzheimer-type pathology, who may represent cases of "preclinical" Alzheimer's disease. ${ }^{20}$ In both groups the preponderance of striatal plaques and cell loss in the substantia nigra may together have contributed to the extrapyramidal features.

We have confirmed previous reports correlating nigral cell loss with disease duration and severity ${ }^{1521}$ especially in patients dying of causes unrelated to Parkinson's disease. This group contained more patients at different stages of disease (mean Hoehn and Yahr score at death 3.6 (range 2-5), mean nigral cell loss score 2.4 (range 1-4)) and thus reflected more broadly the natural history of decline of IPD. In comparison, those dying of parkinsonianrelated causes had more-end stage disease with clear disability and more severe cell loss (mean Hoehn and Yahr score 4.8 (range 4-5), mean nigral cell loss score 3.0 (range $1-4$ ); $p=0.005$ and 0.01 respectively). If the presence of Lewy bodies in brain stem nuclei is considered an essential prerequisite for the diagnosis of Parkinson's disease, then currently used criteria will lead to an $18 \%$ incidence of false positives. Furthermore, although the incidence is unknown, cases with atypical clinical pictures may turn out to have typical Lewy body pathology. This finding throws into question many clinical studies that assume that IPD is a specific morbid entity and may be diagnosed on clinical grounds alone. Although the pathogenesis of Parkinson's disease may prove to be multifactorial further clinico-pathological correlations may help to refine the clinical diagnostic accuracy of Lewy body Parkinson's disease. Until biological markers or more specific neuro-imaging techniques are developed it may have to be accepted that diverse neuropathological disorders including multiple system atrophy and neurofibrillary tangle pathology may produce clinical syndromes indistinguishable from Lewy body Parkinson's disease.

We thank Miss Siobhan Blankson for the histological preparations and Miss Beatrice Vanderstichele for assistance with the manuscript. Dr A J Hughes and Dr S E Daniel are funded by the Parkinson's Disease Society of the United Kingdom.
1 Jellinger $\mathrm{K}$. The pathology of parkinsonism. In: Marsden $\mathrm{CD}$, Fahn S, eds. Movement disorders Vol 2. London Butterworths, 1987:124-65.

2 Jellinger K. New developments in the pathology of Parkinson's disease. In: Streifler MB, Korczyn AD, Melamed E Youdim MBH, eds. Advances in neurology Vol 53. Parkinson's disease. Anatomy, pathology, and therapy. New York: Raven Press, 1990:1-16.

3 Gibb WRG, Lees AJ. The relevance of the Lewy body to the pathogenesis of idiopathic Parkinson's disease. F Neurol Neurosurg Psychiatry 1988;51:745-52.

4 Fearnley JM, Lees AJ. Striatonigral degeneration: a clinicopathological study. Brain 1990;113:1823-42.

5 Sage JI, Miller DC, Golbe LI, Walters A, Duvoisin RC Clinically atypical expression of pathologically typical Lewy body parkinsonism. Clin Neuropharmacol 1990:13:36-47.

6 Joachim CL, Morris JH, Selkoe DJ. Clinically diagnosed Alzheimer's disease: autopsy results in 150 cases. $A n n$ Neurol 1988;24:50-6.

7 Lees AJ. The treatment of multiple system atrophy: striatonigral degeneration and olivopontocerebellar degeneration. In: Bannister R, ed. Autonomic failure: a textbook of clinical disorders of the autonomic nervous system, 2nd ed. Oxford: Oxford University Press, 1988:596-604.

8 Hoehn MM, Yahr MD. Parkinsonism: onset, progression, and mortality. Neurology 1967;17:427-42.

9 Oppenheimer DR. Diseases of the basal ganglia, cerebellum and motor neurons. In: Adams JH, Corsellis JAN, Duchen IW, eds. Greenfield's neuropathology, 4th ed. New York: Wiley, 1984:699-747.

10 Khachaturian ZS. Diagnosis of Alzheimer's disease. Arch Neurol 1985;42:1097-105.

11 Steele JC, Richardson JC, Olszewski J. Progressive supranuclear palsy. Arch Neurol 1964;10:333-59.

12 Kosaka K. Diffuse Lewy body disease in Japan. $f$ Neurol 1990;237:197-204.

13 Parkinson J. An essay on the shaking palsy. London: Sherwood, Nelly and Jones, 1817.

14 Forno LS, Alvord EC. The pathology of parkinsonism. In McDowell FH, Markham CH, eds. Recent advances in Parkinson's disease. Oxford: Blackwell Scientific Publications, 1971:120-61.

15 Bernheimer H, Birkmayer W, Hornykiewicz O, Jellinger K, Seitelberger F. Brain dopamine and the syndrome of parkinsonism and Huntington: clinical, morphological and neurochem.

16 Kuljis RO, Martin-Vasallo P, Peress NS. Lewy bodies in tyrosine hydroxylase-synthesizing neurons of the human cerebral cortex. Neuroscience Letters 1989;106:49-54.

17 Rinne JO, Rummukainen J, Paljarvi L, Sako E, Molsa P Rinne UK. Neuronal loss in the substantia nigra in patients with Alzheimer's disease and Parkinson's disease in relation to extrapyramidal symptoms and dementia. In Iqbal K, Wisniewski HM, Winblad B, eds. Alzheimer's disease and related disorders. New York: Alan $\mathrm{R}$ Liss Inc, 1989.

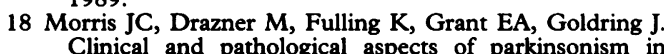
Alzheimer's disease. Arch Neurol 1989;46:651-7.

19 Tyrrell PJ, Rossor MN. Extrapyramidal signs in dementia of the Alzheimer type. Lancet 1989;ii:920.

20 Daniel SD, Lees AJ. Neuropathological features of Alzheimer's disease in non-demented parkinsonian patients. $f$ mer's disease in non-demented parkinsonia

21 Alvord EC Jr, Forno LS, Kusska JA, Kauffman RJ, Rhodes JS, Goetowski CR. The pathology of parkinsonism: a comparison of degenerations in cerebral cortex and brainstem. In: McDowell FH, Barbeau A, eds. Advances in neurology. Vol 5. New York: Raven Press, 1974:175-93. 